\title{
Armadilhas ópticas de dipolo: teoria e experimento de forma didática
}

\author{
Optical dipole traps: theory and experiment didactically
}

\author{
Naomy Duarte Gomes ${ }^{*}\left[0\right.$, Barbara da Fonseca Magnani ${ }^{1}$, Luiz Gustavo Marcassa ${ }^{1}$ \\ ${ }^{1}$ Universidade de São Paulo, Instituto de Física de São Carlos, São Carlos, SP, Brasil.
}

Recebido em 21 de abril de 2020. Revisado em 29 de junho de 2020. Aceito em 04 de julho de 2020.

\begin{abstract}
Armadilhas ópticas de dipolo surgiram a partir do que hoje conhecemos por pinças ópticas. Elas foram inicialmente desenvolvidas para o aprisionamento de átomos neutros, cujo diâmetro é muito menor do que o comprimento de onda da luz utilizada para aprisionar. Devido à sua significância e grande utilização nos experimentos de átomos e moléculas frias, sua compreensão torna-se importante. Portanto, neste trabalho, fazemos, passo-a-passo e didaticamente, a construção teórica da armadilha de dipolo e de um de seus dois possíveis regimes, o regime quase-eletrostático (QUEST). Apresentamos uma possibilidade de montagem experimental, enfatizando as vantagens da armadilha óptica de dipolo no regime QUEST.
\end{abstract}

Palavras-chave: Armadilha óptica de dipolo, QUEST, Polarizabilidade, Átomos frios.

\begin{abstract}
Optical dipole traps emerged as a special case of what today we know as optical tweezers. They were first developed to trap neutral atoms, which posses a diameter much smaller than the wavelength of the trapping light. Due to its significance and applicability in cold atoms and molecules experiments, its comprehension becomes important. Therefore, in this work, we construct, step-by-step and didactically, the theoretical description of the optical dipole trap and one of its two possible regimes, the quasi-electrostatic trap (QUEST). We present one possible experimental setup, emphasizing the advantages of the optical dipole trap in the QUEST regime.
\end{abstract}

Keywords: Optical dipole trap, QUEST, Polarizability, Cold atoms.

\section{Introdução}

O estudo do resfriamento e aprisionamento de átomos neutros tem sido desenvolvido ao longo das últimas décadas e visto grandes resultados, propiciando um melhor entendimento do mundo quântico. Um dos resultados pioneiros foi o trabalho do prêmio Nobel Arthur Ashkin [1], que consistia no aprisionamento de partículas na escala micro por feixes de luz, fazendo uso de forças radiativas para aprisioná-las. Verificou-se que tais forças surgem devido ao espalhamento do campo de luz quando da interação destes com as partículas, e esta armadilha realizada por Ashkin é o que hoje conhecemos por pinças ópticas [2,3].

O trabalho com as pinças ópticas foi estendido para objetos muito menores do que o comprimento de onda da luz de aprisionamento, os átomos. Assim surgiu a primeira armadilha óptica de dipolo, reportada em 1986 por Chu et al [4]. Os trabalhos de Steven Chu envolvendo a armadilha óptica de dipolo também contavam com campos magnéticos que ajudavam no aprisionamento da amostra atômica, uma armadilha que ficou conhecida como Armadilha Magneto Óptica (AMO) 5. Esta armadilha é amplamente utilizada para resfriamento e

*Endereço de correspondência: naomy.gomes@usp.br aprisionamento de átomos, pois permite obter temperaturas muito baixas, da ordem de $n K$, porém a utilização de campos magnéticos causa mudança na estrutura dos níveis de energia atômicos 6].

Em uma armadilha de dipolo, um feixe de luz Gaussiano com comprimento de onda da ordem de centenas de nanometros é focalizado em um conjunto de átomos, cujos diâmetros são da ordem de $0.1 \mathrm{~nm}$. Esta grande diferença entre as dimensões, com o comprimento de onda da luz sendo muito maior que o diâmetro dos átomos, possibilita aproximar o átomo por um dipolo pontual em um campo elétrico, aproximação esta que dá nome à armadilha. A força de dipolo induzido tem mesma direção do gradiente de intensidade da luz, apontando para a região onde a luz é mais intensa.

Existem dois tipos de AOD, dependendo da magnitude da dissonância entre o comprimento de onda do feixe de luz de aprisionamento e o comprimento de onda equivalente à transição atômica considerada. Nestre trabalho, queremos revisar o regime quase-eletrostático da AOD, a armadilha de dipolo do tipo QUEST, que é amplamente utilizada e possui uma descrição teórica de fácil entendimento para alunos de graduação, envolvendo uma formulação que aborda os aspectos clássico e quântico.

Este trabalho é dividido da seguinte forma: na seção 2 revisaremos, passo-a-passo, a formulação matemática 
da AOD considerando o modelo clássico da interação de campos elétricos com distribuições de carga. Na seção 3 . descreveremos o átomo como um sistema quântico e os parâmetros relevantes da armadilha de dipolo, usando a aproximação clássica. A seção 4 considerará a aproximação quase-eletrostática para a armadilha de dipolo. Por fim, a seção 5 abordará as implicações de uma montagem experimental simples.

\section{Interação de um campo elétrico clássico com cargas pontuais}

Um campo elétrico externo (um campo de luz, como no caso aqui descrito) quando interage com uma distribuição de cargas, como átomos, moléculas ou nuvens de átomos, pode causar o deslocamendo de algumas dessas cargas e gerar um momento de dipolo induzido de acordo com a relação:

$$
\mathbf{p}=\alpha \mathbf{E},
$$

onde $\mathbf{p}$ é o vetor momento de dipolo elétrico, definido como

$$
\mathbf{p}=q \mathbf{d}
$$

sendo d o vetor deslocamento de separação das cargas, E o vetor campo elétrico e $\alpha$ a polarizabilidade, a qual não é necessariamente uma quantidade escalar, sendo descrita como um tensor quando a distribuição de cargas é anisotrópica.

\subsection{O modelo de Lorentz}

O modelo de Lorentz é importante para encontrar uma expressão para a polarizabilidade [7]. Neste modelo clássico, tem-se um elétron com massa $m$ muito menor do que a massa do núcleo do átomo. Para o caso de pequenos deslocamentos do elétron em relação ao núcleo, que é estático devido à sua massa muito maior, considera-se que o elétron está preso ao núcleo por meio de uma mola, que se comporta de acordo com a Lei de Hooke: $\mathbf{F}=-k \mathbf{r}$, com constante de mola $k$ e deslocamento r. Quando um campo elétrico é aplicado, a interação campo-elétron induz um momento de dipolo que faz o elétron oscilar.

Considere também, por simplicidade, que o campo elétrico aplicado está arbitrariamente na direção $x$, causa um deslocamento do elétron nesta direção e oscila com frequência $\omega$ de acordo com a relação

$$
E(\omega, t)=\operatorname{Re}\left[E_{0 x} e^{i \omega t}\right] \hat{x},
$$

onde $R e[\ldots]$ representa a parte real de uma quantidade. O momento de dipolo induzido do elétron depende do seu deslocamento $x$ relativo ao núcleo e é dado, de acordo com a equação (2), por $\mathbf{p}=-e x \hat{x}$, onde $-e$ é a carga do elétron e o sinal negativo indica que o elétron se desloca em direção ao núcleo, contrário ao sentido do campo elétrico (Figura 1).

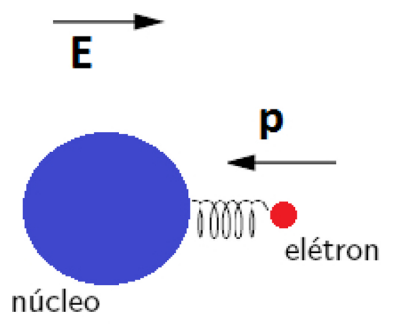

Figura 1: Modelo de Lorentz para um elétron preso ao núcleo por uma mola. Um campo elétrico $\mathbf{E}$ é aplicado no sentido positivo de $x$ e induz um dipolo $\mathbf{p}$ antiparalelo ao campo elétrico.

Levando em conta o caso mais geral, no qual há uma força de amortecimento devido ao campo elétrico, a segunda lei de Newton para o movimento do elétron tornase o tão conhecido oscilador harmônico amortecido [8]:

$$
m \frac{d^{2} x}{d t}+m \Gamma_{\omega} \frac{d x}{d t}+m \omega_{0}^{2} x=-e E
$$

onde $\Gamma_{\omega}$ é a taxa de amortecimento e $\omega_{0}^{2}=k / m$ é a frequência de oscilação da mola, que vem da lei de Hooke. Substituindo $x$ pelo momento de dipolo na equação (4) e rearranjando-a, considerando apenas quantidades escalares, tem-se

$$
\frac{d^{2} p}{d t}+\Gamma_{\omega} \frac{d p}{d t}+\omega_{0}^{2} p=\frac{e^{2} E}{m}
$$

Aqui, pode-se assumir que o momento de dipolo oscila com a mesma frequência do campo elétrico:

$$
p(\omega, t)=\operatorname{Re}\left[\tilde{p}(\omega) e^{i \omega t}\right],
$$

onde $\tilde{p}(\omega)$ pode ser um número complexo.

Substituindo as equações (3) e (6) na equação (5), tem-se:

$$
\begin{gathered}
-\omega^{2} \tilde{p}(\omega) e^{i \omega t}+i \Gamma_{\omega} \omega \tilde{p}(\omega) e^{i \omega t}+\omega_{0}^{2} \tilde{p}(\omega) e^{i \omega t} \\
=\frac{e^{2}}{m} E_{0 x} e^{i \omega t} .
\end{gathered}
$$

Os termos exponenciais da equação (7) são cancelados e o momento de dipolo complexo pode ser isolado:

$$
\tilde{p}(\omega)=\frac{e^{2}}{m} \frac{1}{\omega_{0}^{2}-\omega^{2}+i \Gamma_{\omega} \omega} E_{0 x} .
$$

Comparando as equações (1) e (8), obtém-se, finalmente, a expressão para a polarizabilidade

$$
\alpha(\omega)=\frac{e^{2}}{m} \frac{1}{\omega_{0}^{2}-\omega^{2}+i \Gamma_{\omega} \omega},
$$

onde $\Gamma_{\omega}$ é a taxa de amortecimento clássica devido à perda de energia radiativa, dada por $\Gamma_{\omega}=\frac{e^{2} \omega^{2}}{6 \pi \epsilon_{0} m c^{3}}[9]$, de modo que $e^{2} / m=6 \Gamma_{\omega} \pi \epsilon_{0} c^{3} / \omega^{2}$.

É possível escrever $\Gamma_{\omega}$ como função da taxa de amortecimento na ressonância $\Gamma$ (que é o tempo de vida do 
átomo), por meio da relação $\Gamma_{\omega}=\left(\omega / \omega_{0}\right)^{2} \Gamma$. Portanto, usando a expressão para $e^{2} / m$ e para $\Gamma_{\omega}$, a polarizabilidade pode ser escrita como função de $\Gamma$

$$
\alpha(\omega)=\frac{6 \pi \epsilon_{0} c^{3}}{\omega_{0}^{2}} \frac{\Gamma}{\omega_{0}^{2}-\omega^{2}-i\left(\omega^{3} / \omega_{0}^{2}\right) \Gamma} .
$$

\section{Armadilha óptica de dipolo}

É importante, agora, comparar a descrição quântica do átomo com o modelo clássico. Na visão quântica, o átomo é um sistema com níveis de energia separados entre si por quantidades $\hbar \omega_{0}$ (Figura 2, onde $\omega_{0}$ é a frequência associada à transição entre os níveis considerados. Os níveis de energia excitados possuem um tempo de vida dado pela taxa de decaimento, período a partir do qual o elétron decai espontâneamente do nível excitado. Esta taxa nada mais é do que a taxa de amortecimento na ressonânca $\Gamma$. Esses dois parâmetros, $\omega_{0}$ e $\Gamma$, podem ser obtidos experimentalmente e portanto é desejável expressar quantidades relevantes em função deles.

A primeira quantidade relevante para a AOD é o potencial de dipolo, que fornece a profundidade da armadilha

$$
U_{d i p}=-\frac{1}{2}\langle\mathbf{p E}\rangle=-\frac{1}{2 \epsilon_{0} c} \operatorname{Re}[\alpha(\omega)] I(\mathbf{r}),
$$

onde $I(\mathbf{r})$ é o campo de intensidade da luz, $\langle\ldots\rangle$ denota uma média temporal sobre várias oscilações e o fator $1 / 2$ é adicionado, uma vez que o momento de dipolo é induzido $[10$.

Pode-se também calcular a força de dipolo a partir da equação 11

$$
\mathbf{F}_{d i p}=-\nabla U_{d i p}=\frac{1}{2 \epsilon_{0} c} \operatorname{Re}[\alpha(\omega] \nabla I(\mathbf{r})
$$

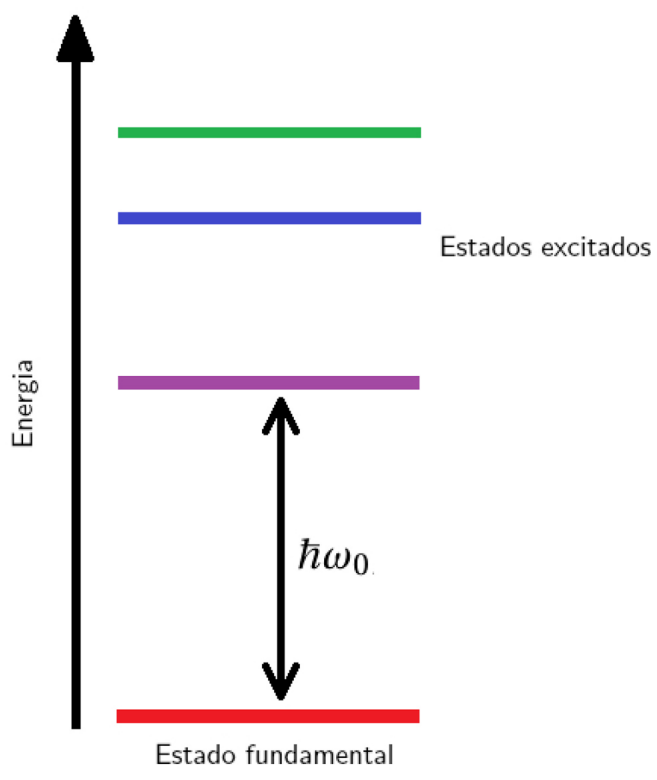

Figura 2: Representação dos níveis de energia em um átomo. que, como pode ser visto pela dependência com o gradiente da intensidade luminosa, aponta na direção onde esta é maior.

A oscilação do dipolo induzido causa absorção de energia do campo de luz, expressa pela potênca absorvida:

$$
P_{a b s}=\left\langle\frac{d \mathbf{p}}{d t} \mathbf{E}\right\rangle=2 \omega \operatorname{Im}[\alpha(\omega)] I(\mathbf{r}),
$$

onde $\operatorname{Im}[\alpha(\omega)]$ representa a parte imaginária da polarizabilidade. A potência absorvida $P_{a b s}$ leva ao segundo parâmetro relevante: a taxa de espalhamento. Esta pode ser vista da seguinte maneira: fótons ressonantes com uma transição energética são absorvidos e, quando emitidos, pelo fato da emissão não possuir direção preferencial, são espalhados. Isso ocorre a uma taxa, chamada de taxa de espalhamento, dada por

$$
\Gamma_{e s p}=\frac{P_{a b s}}{\hbar \omega}=\frac{1}{\hbar \epsilon_{0} c} \operatorname{Im}[\alpha(\omega)] I(\mathbf{r}) .
$$

Considerando a equação 10 para a polarizabilidade e tirando suas partes real e imaginária, pode-se escrever as equações (11) e (14) da seguinte maneira:

$$
\begin{gathered}
U_{d i p}=-\frac{3 \pi c^{2}}{2 \omega_{0}^{3}}\left(\frac{\Gamma}{\omega_{0}-\omega}+\frac{\Gamma}{\omega_{0}+\omega}\right) I(\mathbf{r}), \\
\Gamma_{e s p}=\frac{3 \pi c^{2}}{2 \hbar \omega_{0}^{3}}\left(\frac{\omega}{\omega_{0}}\right)^{3}\left(\frac{\Gamma}{\omega_{0}-\omega}+\frac{\Gamma}{\omega_{0}+\omega}\right)^{2} I(\mathbf{r}) .
\end{gathered}
$$

Nota-se que ambas equações dependem da dissonância $\Delta=\omega_{0}-\omega$.

Observando as equações (15), pode-se considerar dois regimes: um para o qual a dissonância em relação à ressonância atômica seja larga o suficiente, variando de algumas dezenas a algumas centenas de nanometros ( $\omega$ da ordem de $\left.\omega_{0}\right)$, sendo chamado de armadilha longe da ressonância (em inglês, far-off resonance trap - FORT) [11]. O segundo regime apresenta uma dissonância muito grande em relação a qualquer transição atômica, com comprimentos de onda da luz de aprisionamento ao redor de $10 \mu \mathrm{m}$, sendo chamado de armadilha quase-eletrostática (em inglês, quasi-electrostatic trap - QUEST) [12]. Para este segundo regime, da armadilha QUEST, as equações 15 podem ser simplificadas, como será visto na próxima seção.

\section{QUEST, a armadilha quase-eletrostática}

Quando a frequência $\omega$ do campo elétrico da luz é muito menor do que qualquer frequência de transição $\omega_{0}$ (ou, em termos de comprimento de onda, quando o da luz é muito maior do que qualquer comprimento de onda de transição atômica), é como se o átomo não visse nenhuma mudança no campo da luz, de modo que pode-se considerar um regime quase-eletrostático. Nessa condição, onde $\omega \ll \omega_{0}$, $\alpha(\omega)$ pode ser substituído pela polarizabilidade estática 
$\alpha_{\text {estat }} \equiv \alpha(\omega \rightarrow 0)$. Fazendo uma expansão em série de Taylor da equação (9) ao redor de $\omega=0$, obtém-se $\alpha_{\text {estat }}=e^{2} / m \omega_{0}^{2}$, que é uma constante para cada átomo considerado. Levando em conta esta aproximação quaseeletrostática, a equação (11) fica

$$
U_{d i p}=-\frac{\alpha_{\text {estat }}}{2 \epsilon_{0} c} I(\mathbf{r}) \text {. }
$$

É importante notar que o potencial da AOD no regime QUEST é sempre atrativo e não depende da dissonância em relação a qualquer condição de ressonância. Portanto, a QUEST pode armadilhar átomos em qualquer nível de energia simultaneamente usando apenas feixes de luz, diferentemente da FORT.

No que diz respeito à taxa de espalhamento em uma QUEST, a equação $15 \mathrm{~b}$ mostra que $\Gamma_{\text {esp }}(\omega \rightarrow 0) \rightarrow 0$, posto que há um fator $\omega^{3}$ no nominador. Essa ausência de espalhamento de fótons significa que não há luz ressonante para excitar os átomos e, portanto, estes se encontram em seus estados fundamentais. Deste modo, o $\alpha_{\text {estat }}$ considerado será a polarizabilidade do estado fundamental. Além disso, $\Gamma_{e s p}(\omega \rightarrow 0) \rightarrow 0$ também implica que o aquecimento devido à emissão espontânea é negligível.

\section{Implementação experimental}

A equação 16 mostra que a profundidade da armadilha depende da intensidade da luz, portanto, feixes de alta potência no espectro infravermelho distante, como o caso de lasers de $\mathrm{CO}_{2}$, devem ser empregados. Valores usuais de potências utilizadas variam de $5 \mathrm{~W}$ a $40 \mathrm{~W}$.

Para a QUEST, comumente são utilizados feixes Gaussianos. O perfil de intensidade de um feixe Gaussiano é dado por 13.

$$
I(x, y)=\frac{2 P}{\pi w^{2}(x)} \exp \left(\frac{-2 y^{2}}{w^{2}(x)}\right),
$$

onde $P$ é a potência do feixe, $y$ é a coordenada vertical e $w(x)$ é a cintura do feixe ao longo da direção de propagação $x$, dada por

$$
w(x)=w_{0} \sqrt{1+\left(\frac{x}{x_{R}}\right)^{2}} .
$$

Os parâmetros $\lambda, w_{0}$ e $x_{R}=\pi w_{0}^{2} / \lambda$ denotam, respectivamente, o comprimento de onda da luz, a cintura mínima do feixe Gaussiano e o alcance de Rayleigh 14 .

Com as equações (17) e (18), é possível escrever o potencial da equação (16) como função da cintura do feixe e da potência

$$
U(x, y)=-\frac{\alpha_{\text {estat }}}{\epsilon_{0} c} \frac{P}{\pi w^{2}(x)} \exp \left(\frac{-2 y^{2}}{w^{2}(x)}\right) .
$$

A Fig. 3 mostra os gráficos do potencial da equação 19. no eixos $x$ e $y$, para $y=0$ e $x=0$, respectivamente.
O valor máximo da profundidade do potencial de aprisionamento na QUEST é dado pela equação 19 quando $x=y=0$

$$
U_{0}=-\frac{\alpha_{e s t a t}}{\epsilon_{0} c} \frac{P}{\pi w_{0}^{2}} .
$$

Bons valores de profundidade máxima da armadilha, que são medidos como temperatura por meio da relação $\left|U_{0}\right| / k_{B}$, sendo $k_{B}$ a constante de Boltzmann, devem ser no regime de mili ou micro Kelvin. Este parâmetro, $\left|U_{0}\right| / k_{B}$, deve ser comparado à temperatura $T$ dos átomos que serão transferidos para a AOD. Se $T \leq\left|U_{0}\right| / k_{B}$, os átomos permanecem aprisionados; caso contrário, os átomos podem escapar da armadilha, pois são muito energéticos para ficarem presos pelo potencial.

Uma configuração experimental simples para uma AOD é composta de um feixe Gaussiano colimado passando por uma lente de distância focal $f$, cujo foco se encontra onde os átomos estão concentrados (veja Figura 4. Nessa configuração, o valor mínimo de cintura pode ser calculado como

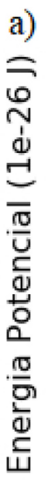
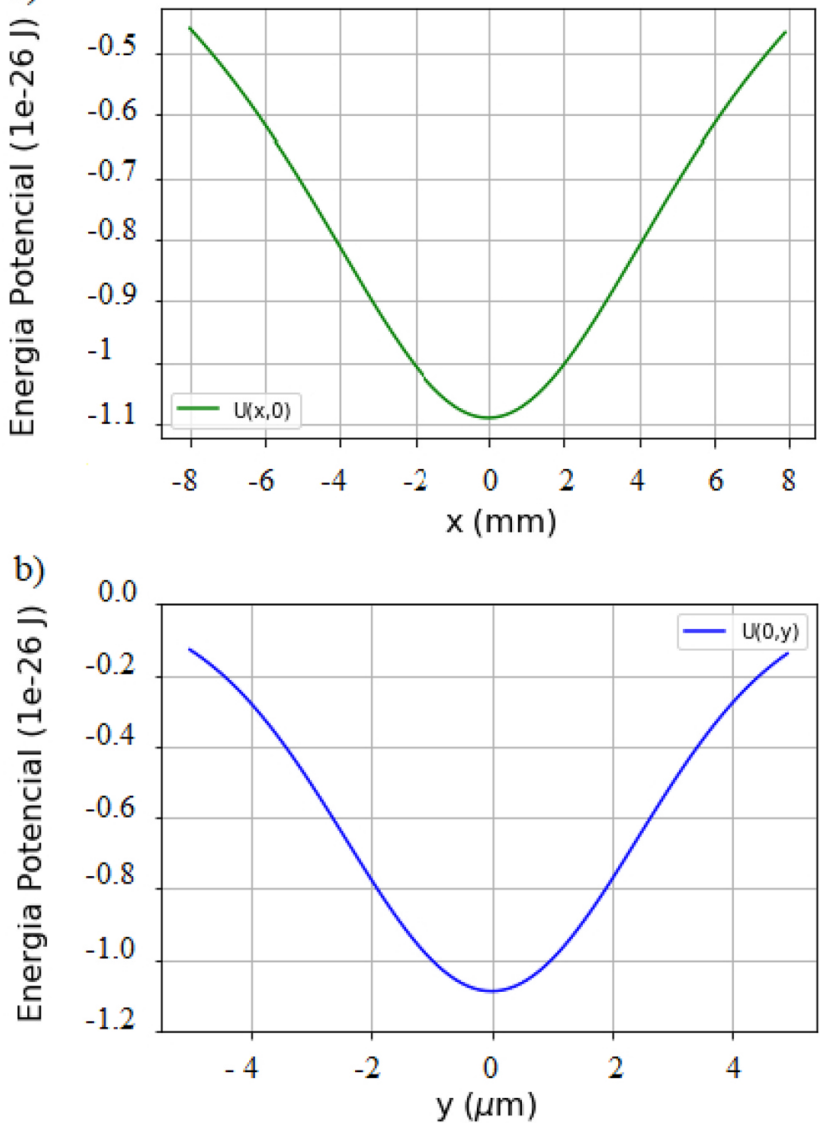

Figura 3: Gráficos do potencial $\mathrm{U}(\mathrm{x}, \mathrm{y})$ para a) eixo $\mathrm{x}$, sendo $y=0 \mathrm{e}$ b) eixo $\mathrm{y}$, sendo $x=0$. Os parâmetros utilizados nestes gráficos foram: $P=40 \mathrm{~W}$, $\alpha_{\text {estat }}$ do Rubídio e cintura $w_{0}=48 \mu m$. 


$$
w_{0}=\frac{\lambda f}{\pi w} .
$$

É importante salientar o papel da força da gravidade na AOD. Sendo sua direção vertical para baixo, conforme mostra a Figura 4 . ela faz com que o potencial na direção vertical seja diminuído por um termo mgy, ou seja, o potencial será dado por $U(x . y)-m g y$, onde $m$ é a massa total dos átomos aprisionados e $g$ a gravidade. A Figura 5 compara o potencial de aprisionamento na direção vertical com e sem o efeito da gravidade, mostrando a perda de simetria na profundidade deste potencial quando a gravidade é considerada, formando um "escorregador"de átomos. Este efeito deve ser levado em conta quando da montagem de uma AOD para que os átomos sejam aprisionados com eficácia.

Apesar da simplicidade experimental de uma AOD no regime da QUEST, a equação 16 mostra que esta armadilha não é capaz de resfriar, uma vez que o potencial é puramente conservativo e não há, portanto, dissipação de energia. Deste modo, átomos aprisionados em uma QUEST requerem um resfriamento posterior para aumentar a densidade atômica da amostra aprisionada,

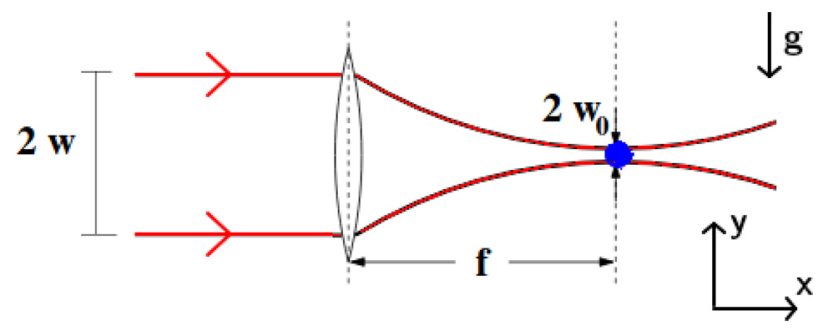

Figura 4: Representação esquemática de uma armadilha de dipolo: um feixe é focalizado horizontalmente a uma distância $f$. Em azul, estão representados os átomos presos no foco do feixe. A gravidade no desenho é vertical para baixo.

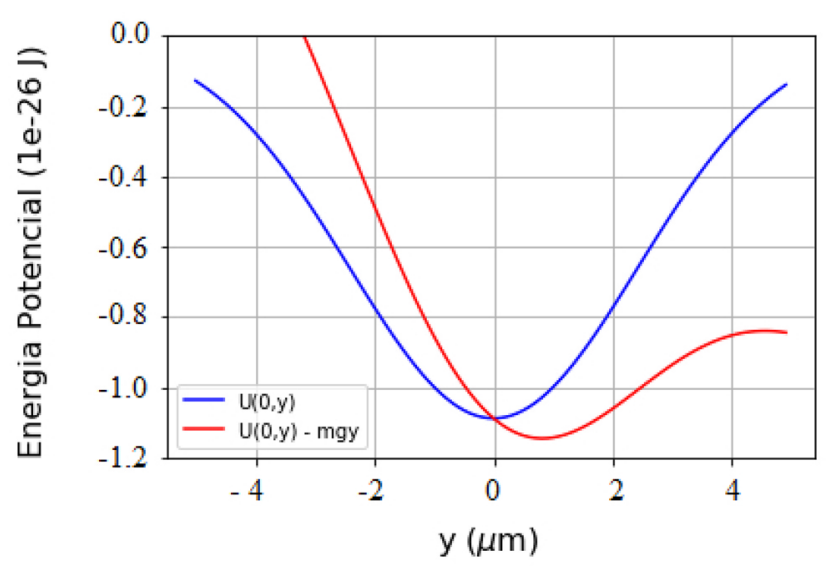

Figura 5: Comparacão dos gráficos do potencial $U(x, y)$ para o eixo y com (em vermelho) e sem (em azul) o efeito da gravidade. Esta faz com que o potencial perca simetria e apresente um formato de um "escorregador"de átomos. Os parâmetros utilizados neste gráfico foram: $P=40 W, \alpha_{\text {estat }}$ do Rubídio, massa do Rubídio 87 e cintura $w_{0}=48 \mu \mathrm{m}$. uma vez que, quanto maior essa densidade, mais fácil é o aparecimento dos efeitos quânticos. Uma técnica de resfriamento que pode ser empregada é o resfriamento evaporativo 15, 16]. Um exemplo é o trabalho de Barret et al [17], no qual foi aplicado um resfriamento evaporativo a uma QUEST de $\lambda=10.6 \mu \mathrm{m}$, através de uma diminuição gradual na potência do laser, permitindo a obtenção de um condensado de Bose-Einstein [18].

\section{Conclusão}

A armadilha óptica de dipolo surgiu como uma opção para o aprisionamento de átomos neutros, em um regime no qual a aproximação de dipolo pudesse ser aplicada. Ela é experimentalmente simples, uma vez que requer apenas um feixe de laser ou feixes de laser cruzados com foco na amostra atômica.

No regime da QUEST, a AOD apresenta vantagens experimentais e simplicidade de descrição teórica, sendo de mais fácil compreensão para iniciantes do que a FORT, uma vez que, na QUEST, os cálculos são simplificados com a aproximação eletrostática. Além disso, a QUEST apresenta outras duas vantagens importantes frente à FORT, tais como um aprisionamento que independe dos níveis de energia atômico, o que permite armadilhar átomos em qualquer estado, além do aquecimento devido ao espalhamento dos fótons ser desprezível.

Apesar de suas vantagens, as AODs não podem ser usadas para resfriar átomos, devido ao potencial conservativo. Por esse motivo, faz-se necessário posterior resfriamento. De qualquer forma, estas armadilhas são muito utilizadas em diversos experimentos com átomos frios, de átomos de Rydberg 19 a condensados de BoseEinstein, sendo, portanto, de grande importância seu entendimento.

\section{Referências}

[1] A. Ashkin, Phys.Rev.Lett. 25, 1321 (1970).

[2] A. Ashkin, Phys. Rev. Lett. 24, 4 (1970).

[3] Ashkin A e Dziedzic JM, Science 235, 4795 (1987).

[4] S. Chu, J.E. Bjorkholm, A. Ashkin e A. Cable, Phys. Rev. Lett. 57, 314 (1986).

[5] E.L. Raab, M. Prentiss, A. Cable, S. Chu e D.E. Pritchard, Phys. Rev. Lett. 59, 2631 (1987).

[6] P. Zeeman, Nature 55, 347 (1897).

[7] A.F.J. Levi, The Lorentz oscillator model (Morgan and Claypool Publishers, Williston, 2016).

[8] H.M. Nussenzveig, Curso de Física Básica (Blucher, Rio de Janeiro, 2017).

[9] J.D. Jackson, Classical Electrodynamics (John Wiley and Sons, New York, 1962).

[10] D. Halliday, R. Resnick e J. Walker, Fundamentos de Física - Eletromagnetismo (Editora LTC, São Paulo, 2011), v. 3.

[11] J.D. Miller, R.A. Cline e D.J. Heinzen, Phys. Rev. A 47, R4567 (1993). 
[12] T. Takekoshi, J.R. Yeh e R.J. Knize, Opt. Comm. 114, 421 (1995)

[13] F. Pampaloni e J. Enderlein, arXiv:physics/0410021 (2004).

[14] A.E. Siegman, Lasers (University Science Books, Mill Valley, 1986).

[15] W. Ketterle e N.J. Van Druten, Adv. At. Mol. Opt. Phys 37, 181 (1996).

[16] S. Chaudhuri, S. Roy e C.S. Unnikrishnan, Phys. Conf. Ser.80, 012036 (2007).

[17] M.D. Barrett, J.A. Sauer e M.S. Chapman, Phys. Rev. Lett. 87, (2001).

[18] M. Anderson, J. Ensher, M. Matthews, C. Wieman e E. Cornell, Science 269, 198 (1995).

[19] L.F.B.F. Gonçalves, Construção de uma armadilha de dipolo tipo QUEST para átomos de Rydberg. Dissertação de Mestrado, Universidade de São Paulo, São Paulo (2012). 